\title{
Intervención psicológica en un deportista con Fibrilación auricular: Un estudio de caso único
}

\section{Psychological intervention in an athlete with atrial fibrillation: $A$ unique case study} \\ Consulta Privada. \\ Colegio Oficial de Psicólogos Comunidad Valenciana.
}

\section{resumen/alsstract:}

Se presenta una intervención aplicada con un deportista diagnosticado de Fibrilación Auricular que desarrolla a posteriori un trastorno de ansiedad con rasgos hipocondriacos. El eje central del trastorno en este caso es la presencia de palpitaciones y su retroalimentación mediante las conductas de evitación y comprobación propias del cuadro de ansiedad. Por ello, la intervención se realizó integrando técnicas propias de la psicología del deporte, la psicoterapia breve estratégica y la psicoterapia cognitiva. Se aplicó una metodología de investigación de caso único con medidas previas y posteriores a la intervención (test - retest), utilizando para ello los cuestionarios de Bienestar psicológico de Ryff y una versión abreviada del ISRA para medir los resultados, así como autoregistros y otras medidas cualitativas propias del modelo de aplicación.

Los resultados muestran una mejora en todas las puntuaciones obtenidas tras la intervención y en los criterios cualitativos utilizados,mostrando la eficacia de la intervención programada y la consecución de los objetivos iniciales.

An intervention is presented a case about an athlete whose diagnosis was atrial flutter who subsequently develops an anxiety disorder with hypochondriacal features. The central axis of the disorder in this case is the presence of heart palpitations and their feedback through avoidance and checking behaviors typical of the anxiety picture.

For this reason, the procedure was made integrating techniques from sports psychology, brief therapy approaches and cognitive psychotherapy. A single case research methodology was applied with pre and post intervention measures (test - retest).

In addition, employing Ryff's psychological well-being questionnaires and an ISRA abbreviated version which were used to measure the results, as well as self-records and other qualitative gauges characteristics of the application model.

Finally, the results reveal an improvement in all the scores achieved after the intervention and qualitative criteria used, demonstrating the effectiveness of the scheduled procedure and the achievement of the initial goals.

\section{palabras clave/keywords:}

intervención psicológica, psicología del deporte, fibrilación auricular, Terapia breve estratégica, hipocondría, palpitaciones.

Psychological procedures, sports psychology, atrial flutter, brief therapy approaches, hypochondria, heart palpitations. 


\section{Introduccion}

En el ámbito de la consulta privada de un psicólogo, con frecuencia se presentan casos que pueden demandar la integración de diferentes enfoques terapéuticos y el uso de técnicas extraídas de diversos ámbitos de aplicación, debido a que la sintomatología puede ser variada y abarcar diferentes planos de la salud física y mental. Por ello, la intervención profesional en este ámbito debe ser eficiente en tiempo y resultados, y para ello debe elaborarse un plan con objetivos bien definidos que permita medir la calidad de la intervención.

A continuación de describe el programa intervención en uno de estos casos, haciendo uso de técnicas y estrategias propias de la Psicología de la Salud, la Psicoterapia y la Psicología del Deporte (COP, 1998). En concreto se refiere a un deportista diagnosticado con un trastorno del ritmo cardiaco, denominado Fibrilación Auricular (FA), tipo vagal, (Mitchell, 2017) caracterizado por la pérdida de sincronía del ritmo cardiaco, donde este deja ser regular y constante y el tiempo entre latidos es desigual, produciéndose taquicardia (aumento de la frecuencia a la que se contrae el corazón) en periodos de reposo. Se ha demostrado una relación epidemiológica entre la aparición de este trastorno (FA) y la realización de entrenamiento de resistencia de alta intensidad y volumen, considerándose a este nivel que no supone un factor de riesgo cardiovascular o en el desarrollo de patología cardiaca (Nadal, Elosua y Mont, 2016). Sin embargo, el propio diagnóstico y la reacción psicológica de miedo y temor asociada al mismo, provocó la aparición de ansiedad, pánico e ideas obsesivas en torno a la propia salud que a su vez retroalimentó la sintomatología del FA, generándose un círculo vicioso de tal magnitud que el cliente se estaba planteando el abandono de toda actividad deportiva (y laboral) cuando solicita ayuda profesional.

La hipótesis principal parte de la consideración de las palpitaciones como un síntoma retroalimentado por la ansiedad, pues si bien el inicio de las mismas se debe a la FA, en el momento de acudir a consulta (cinco meses después del diagnóstico)se evidencian conductas claras de evitación, comprobación y vigilancia que muy probablemente explican su aparición con mayor frecuencia.

La intervención se desarrolló desde el marco de la Terapia Breve Estratégica (en adelante TBE) de Watzlawick y Nardone $(1993,2014)$, un enfoque de terapia breve sistémica enfocado en la ruptura del círculo vicioso que se produce como consecuencia de las soluciones intentadas del paciente, al que se suman técnicas clásicas en las intervenciones propias del ámbito de la psicología del deporte, tales como el establecimiento adecuado de objetivos (Locke y Latham, 1984)o el entrenamiento en atención y concentración (Weinberg y Gould, 1995) y otras comunes al ámbito de la psicoterapia, tales como las estrategias y técnicas cognitivas procedentes del modelo de Beck (Clark y Beck, 2012).

La intervención se desarrolló a lo largo de 10 sesiones y para medir se utilizaron dos escalas, la de Bienestar Psicológico de Ryffy una versión reducida del cuestionario ISRA (Inventario de situaciones y respuestas de ansiedad), además de preguntas de escala y autoregistros propios del modelo de intervención. 


\section{Descripción del caso}

El participante es un varón de 39 años de edad, soltero, cuya profesión habitual es instructor de educación física (fitness, Cardio box, Step, etc.)y deportista aficionado (duatlón, larga distancia) que a raíz de una prueba rutinaria de esfuerzo y varias visitas a médicos especialistas es diagnosticado cinco meses antes de un trastorno del ritmo cardiaco (FA), caracterizado por la presencia de taquicardias en periodos de reposo. En base a este diagnóstico aparece diversa sintomatología concurrente con un trastorno de ansiedad, tal como autovigilancia y autocomprobaciones, preocupación por el propio estado de salud, miedo a sufrir un ataque cardiaco y morir, evitación de actividades habituales (laborales, deportivas e incluso sociales), llegando a producirse ataques de pánico puntuales, que a su vez retroalimentan la frecuencia de aparición de las palpitaciones. Este proceso dura aproximadamente 5 meses hasta que decide buscar ayuda psicológica profesional motivado por su cardiólogo de referencia, que le intenta tranquilizar acerca de la escasa gravedad de su patología cardiaca y le insiste en la idea de que las palpitaciones son "autoprovocadas" en su mayor parte, pudiendo continuar el paciente con su estilo de vida habitual.

Informa además que anteriormente estaba muy bien en su trabajo y su estilo de vida vinculado al deporte, la alimentación saludable, el bienestar, etc. que, si bien ocupaba gran parte de su tiempo, le resultaba muy satisfactorio. No obstante en el momento de acudir a consulta, ha reducido significativamente su carga laboral como instructor de actividades deportivas, así como sus salidas de ocio, y abandonado por completo la práctica y competición del duatlón. Se encuentra además muy angustiado por la posibilidad de un ataque al corazón y sus consecuencias, por lo que se plantea seriamente abandonar su profesión habitual, y controla constantemente su frecuencia cardiaca mediante un pulsómetro de muñeca.

\section{Intervención.}

Una premisa básica del enfoque estratégico es trabajar desde la perspectiva de una intervención breve y focalizada, orientada hacia la extinción de los síntomas, por una parte, y hacia la reestructuración de la percepción que la persona tiene de sí misma, de los otros y del resto del mundo, por otra parte. En otras palabras, el objetivo principal de un terapeuta estratégico es el de resolver rápidamente los problemas y trastornos que pueden convertirse en invasivos e invalidantes (Nardone, 2003 y 2004).

En este caso la intervención se limitó a 10 sesiones, asumiendo como objetivos los planteados por el cliente en la primera sesión y usando una metodología de test -retest tanto con medidas cuantitativas (Bienestar psicológico y Ansiedad) como cualitativas (Ausencia vs presencia de los objetivos planteados), tomándose las medidas al principio y al final de la intervención.

\section{Evaluación}

Para las medidas test -retest de la intervención se utilizaron las siguientes escalas y cuestionarios:

Escala de Bienestar Psicológico de Ryff en su versión española reducida de Díaz, Rodrí- 
guez-Cavajal, Blanco, Moreno-Jiménez, Gallardo,Valle y Van-Dierendonck(2006). Esta escala está formada por 29 ítems medidos en una escala de seis puntos de tipo Likert; donde 1 es "completamente en desacuerdo" y 6 es "completamente de acuerdo" y se agrupan en seis dimensiones que presentaron una consistencia y análisis confirmatorio del modelo en esta escala de: Autoaceptación $(\alpha=.84)$ que mide la actitud de la persona hacia sí misma, Dominio del entorno $(\alpha=.82)$ que hace referencia a los entornos que crea la persona para facilitar el cumplimiento de sus objetivos, Relaciones positivas $(\alpha=.78)$ que se refiere a la capacidad de la persona para mantener relaciones sanas y basadas en la confianza, Propósito en la vida $(\alpha=.70)$ que mide la concreción de las metas de la persona, Crecimiento personal $(\alpha=.71)$ que es la capacidad de la persona para buscar situaciones que le permitan autorealizarse personalmente, y Autonomía $(\alpha=.70)$ que hace referencia a si la persona es capaz de separar y mantener su identidad personal de la social.

Test de autoevaluación de la Ansiedad. Versión reducida de 12 ítems del cuestionario ISRA (Inventario de situaciones y respuestas de ansiedad) de Miguel-Tobal y Cano-Vindel (2012), publicado en la Web de la Sociedad Española para el Estudio de la Ansiedad y el Estrés (SEAS).Cuantifica las respuestas o síntomas de ansiedad en base a tres sistemas de respuesta, descritos como Respuestas Cognitivas, Respuestas Fisiológicasy Respuestas Motoras, arrojando un índice global de Ansiedad.

Se utilizaron también autorregistros de la aparición de palpitaciones, debiéndose anotar en ellas los pensamientos asociados y la respuesta conductual aplicada.

La evaluación cualitativa se realizó en base a la definición mutua con el paciente de los objetivos de la intervención, utilizando para ello preguntas específicas para constatar su presencia al final de la intervención. El resumen de los objetivos y el método para evaluar su consecución se muestran en la tabla 1 .

Tabla 1.- Objetivos de la intervención y método de evaluación

Objetivo

"Volver a estar como antes del diagnóstico"

"No tener inseguridad, ansiedad o miedo"

"Salir más y disfrutarlo"

"Volver a tener la misma carga laboral"

"Volver a hacer duatlón y competir"

"No estar obsesionado con mi pulso "

"No estar preocupado por mi salud"
Medida

Bienestar Psicológico (Ryff)

Grado de Ansiedad (ISRA)

Conductual - Autoinformado

Conductual - Autoinformado

Conductual - Autoinformado

Cognitivo - Número/intensidad

Cognitivo - Presencia/intensidad 


\section{Intervención}

A continuación se describen las sesiones realizadas, el objetivo de las mismas dentro del modelo de intervención y las principales técnicas utilizadas junto con una breve descripción de su función y la evolución del participante. El resumen de las técnicas empleadas, su modelo de referencia y su objetivo terapéutico pueden encontrarse en la tabla 2.

Tabla 2.- Resumen de técnicas empleadas en la intervención, modelo de procedencia y objetivo.

\begin{tabular}{lll} 
Tecnicas & Modelo Procedencia & Objetivo \\
\hline FASE EVALUACIÓN-Sesiones 1 a 2 & & \\
Diálogo Estratégico & TBE & Evaluar/ Reestructurar \\
Pregunta Milagro & TBE & Evaluar \\
Establecimiento de Objetivos & Psicología del Deporte & Evaluar/ Reestructurar \\
Cuestionario Ryff & Común & Evaluar \\
Cuestionario Ansiedad /vr ISRA & Común & Evaluar \\
Autorregistro Ansiedad & Cognitivo/Conductual & Evaluar
\end{tabular}

FASE INTERVENCIÓN - Sesiones 3 a 5

Silla rumiatoria/Peores fantasías TBE

Reestructurar respuesta emocional/ cognitiva

Exposición a situaciones ansiógenas Común

Focalización

Reinterpretación sensaciones físicas

Medidas fisiológicas

Estructuración actividades cotidianas y Común

laborales

Psicología del Deporte

Psicología del Deporte

Psicología del Deporte

FASE ESTABILIZACIÓN - Sesiones 5 a 8

Diálogo estratégico

TBE

Establecimiento de objetivos

Asertividad

Psicología del Deporte

Común

Exposición a situaciones ansiógenas

Autoinstrucciones

FASE SEGUIMIENTO -Sesiones 9 a 10

Establecimiento de Objetivos

Cuestionario Ryff

Cuestionario Ansiedad /vr ISRA

Cómo Empeorar

Prescribirse la Fragilidad
Común

Psicología del Deporte
Psicología del Deporte

Común

Común

TBE

TBE
Reestructurar respuesta emocional/ cognitiva

Control de la atención

Control de la atención

Eliminar autovigilancia

Aumentar percepción de control

Reestructurar

Evaluar/ Reestructurar

Relaciones Familiares

Reestructurar respuesta emocional/ cognitiva

Motivación/Control

Atencional
Evaluar/ Reestructurar

Evaluar

Evaluar

Prevención recaídas

Prevención recaídas/

Reestructurar 
- Sesiones 1 y 2: Fase Evaluación. (12/04/18 - 19/04/18)

Objetivos: Creación marco relacional. Evaluación de la demanda y los objetivos de la intervención. Aplicación de los cuestionarios (Bienestar Psicológico y Ansiedad), medidas iniciales. Explicación y administración de autoregistros para situaciones de ansiedad.

En esta primera fase, se realiza la recepción del cliente y se generan las bases, desde el diálogo estratégico, para redefinir y establecer la causalidad de la dinámica del problema. A partir de entonces, y a través de la técnica de la "pregunta milagro" (Beyebach y Herrero de Vega, 2016) y la de establecimiento de objetivos de Locke y Latham (1984), se definen los objetivos de la intervención y la forma de medirlos, de modo que ambos (Psicólogo y cliente), podamos constatar si la intervención ha sido o no efectiva. Los cuestionarios se dirigen a objetivar determinados aspectos de esta primera evaluación, y el autorregistro se prescribe para entrar en el detalle de las situaciones y experiencia emocional y cognitiva del cliente en situaciones ansiógenas en el momento.

- Sesiones 3 a 5: Fase Intervención. Reducción de comprobaciones, autovigilancia y otros síntomas de ansiedad. (25/04/18 - 7/06/18).

Objetivos: Reducir los síntomas de ansiedad y conductas asociadas: minimizar evitación de situaciones ansiógenas, eliminar comprobaciones constantes de pulso/palpitaciones, reinterpretación de sensaciones físicas, reducción y reestructuración de los pensamientos de preocupación sobre la salud/enfermedad.

En esta fase, las sesiones de establecen de forma bisemanal. La primera tarea terapéutica fue restructurar y limitar las tareas de comprobación del pulso mientras se expone al sujeto progresivamente a las tareas deportivas propias de su ámbito laboral, deportivo y de ocio.

La premisa que se le prescribe es configurar el pulsómetro para emitir una alarma cuando se superen las 180 pulsaciones/minuto, un "margen de seguridad" establecido por su cardiólogo muy razonable teniendo en cuenta la forma física y edad del sujeto cuando realiza actividad física o deportiva. En caso de sonar la alarma, debe detenerse y respirar abdominalmente para recuperar su ritmo cardiaco y respiratorio normal. En cualquier otro supuesto (momentos de ocio o sin actividad física) debe, literalmente, desconectar y guardar el pulsómetro en un cajón, y focalizar su atención en las tareas que está realizando. Se trabajan para ello técnicas de focalización y reinterpretación de las sensaciones físicas, de forma que el cliente obtenga mayor control cognitivo de sus pensamientos y preocupaciones sobre la salud, reforzándolo con reestructuraciones in situ durante las sesiones para controlar el proceso.

En la misma línea, se prescribe la técnica de la "silla rumiatoria" (Beyebach y Herrero de Vega, 2016) limitada a 30 minutos al día, para gestionar los pensamientos intrusivos. Se completa la línea de intervención con una racionalización de los horarios de trabajo, ocio, deporte y descanso dirigido a focalizar la atención en una rutina y potenciar la percepción de control, modificando fuentes residuales de estrés.

- Sesiones 5 a 8: Regulación/ Estabilización. (7/06/2018 - 26/07/2018)

En esta fase, se constata una reducción muy significativa de la sintomatología de ansiedad (palpitaciones, pensamientos intrusivos, preocupaciones y autovigilancia), dando lugar de 
forma natural a una transición en los objetivos a mantener y estabilizar el bienestar recuperado, y potenciar otros aspectos de la vida del cliente inicialmente no descritos como parte de la problemática pero que quizá son fuentes de estrés y ansiedad para él, incluidas sus relaciones familiares y de pareja y su necesidad de autorrealización. Dentro de este último aspecto, se establecen nuevos objetivos en la recuperada práctica deportiva de Duatlón y se realiza un entrenamiento en autoinstrucciones (Buceta, 1998) para potenciar el rendimiento en este ámbito.

\section{Seguimiento}

- Sesiones 9 a 10: Seguimiento y prevención de recaídas (13/09/18 - 18/10/18)

Se dedica estas últimas sesiones, con frecuencia mensual, a consolidar los cambios obtenidos, mantener los procesos de reestructuración y prevenir la aparición de recaídas. Además de ello, se toman las medidas finales y se verifica el grado de cumplimiento de los objetivos iniciales y de los surgidos en la fase de Estabilización.

\section{Resultados}

A continuación, en la tabla 3 y la figura 1 se muestran los resultados obtenidos en las fases previa y posterior a la intervención, medidos con la escala de Bienestar Psicológico de Ryff y la versión abreviada del ISRA.

Como se puede observar en la Figura 1, todas las dimensiones de la escala de Bienestar Psicológico incrementan su puntuación, indicando un cambio cuantitativo en las escalas de Autoaceptación, Autonomía, Crecimiento personal, Propósito en la vida y en el Índice global de Satisfacción, al cambiar la puntuación de estar por debajo de la media a estar por encima de la media de referencia tras la intervención. La dimensión Relaciones Positivas aumenta también su puntuación para situarse en posiciones cercanas aunque inferiores a la media. La dimensión Dominio del entorno permanece por encima de la media e incrementa su puntuación. Todo ello se refleja en un incremento muy considerable en el índice de Bien-

Tabla 3.- Resultados en medidas Pre y Post intervención con medias.

\begin{tabular}{lccc}
\hline Escala & PRE & POST & MEDIA \\
HOMBRES \\
Autoaceptación & 13 & 31 & 25,5 \\
Relaciones positivas & 15 & 24 & 25,9 \\
Autonomía & 30 & 36 & 33,8 \\
Dominio del entorno & 26 & 34 & 25,6 \\
Crecimiento personal & 29 & 36 & 31,8 \\
Propósito de Vida & 15 & 29 & 26,9 \\
Ansiedad & 20 & 8 & 16 \\
Satisfacción Global & 128 & 190 & 138 \\
\hline
\end{tabular}


Figura 1.- Resultados en medidas Pre y Post intervención con medias en Bienestar y Ansiedad

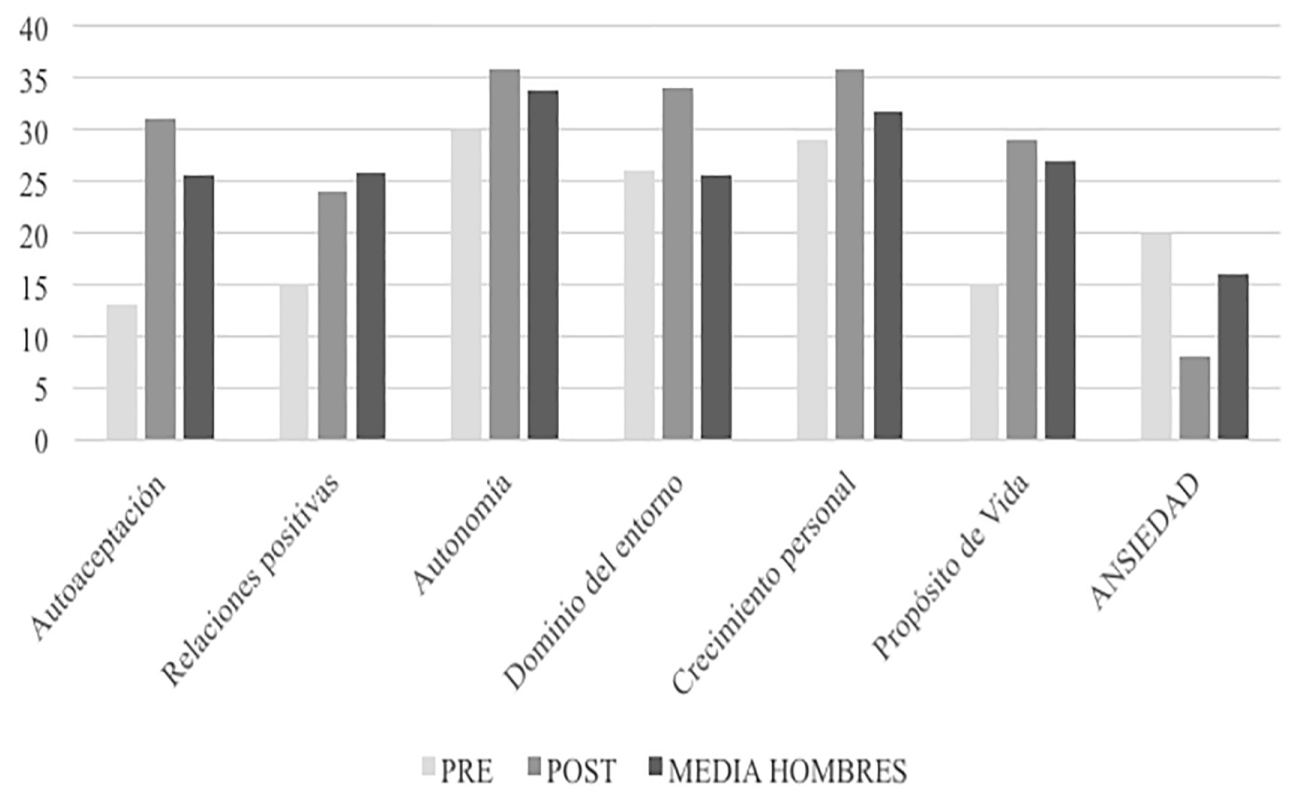

estar Global, pasando de estar por debajo de la media de referencia en la fase inicial (128 puntos) a situarse muy por encima de esta media de referencia al final de la intervención (190 puntos).

En cuanto a la escala de Ansiedad, se produce una reducción importante de la puntuación, cambiando su posición inicial por encima de la media a hallarse muy por debajo de esta al final de la intervención.

Como dato ilustrativo, el ítem "Palpitaciones/ Aceleración cardiaca", síntoma importante de la dinámica problema, fue valorado con la puntuación máxima al inicio de la intervención, definida como "casi siempre". En la medida al final de la intervención pasa a nominarse como "pocas veces", lo cual es un buen reflejo de su extinción como respuesta de ansiedad.

Retomando los objetivos de la fase inicial planteados en la tabla 1, el participante informa que efectivamente casi todos han sido conseguidos al final de la intervención, permaneciendo únicamente de forma parcial, con menor intensidad y frecuencia de aparición que al principio, las preocupaciones y pensamientos en torno a su estado de salud y forma física, lo cual se constata a través de los autorregistros.

Además informa de otras mejoras no incluidas en la fase de evaluación como objetivos, tales como una mejora en las relaciones familiares, definiendo la comunicación como "más asertiva/menos manipuladora", y una buena estabilidad y satisfacción en su relación de pareja. 
Con respecto al análisis cualitativo, en el seguimiento telefónico transcurrido un año desde la última sesión, el participante informa que la sintomatología de ansiedad no ha vuelto a aparecer en ningún momento, considerándose resuelto el motivo inicial de consulta, y confiando en sus recursos de afrontamiento ante situaciones estresantes. Se muestra además muy satisfecho tanto en el ámbito profesional como en el deportivo, informando de nuevos retos en dicho área, tales como estar entrenado para realizar un Maratón. Confirma que sus relaciones familiares y de pareja se mantienen razonablemente bien, aunque ha sufrido alguna pérdida significativa. Considera no obstante que su estado de ánimo es bueno y se muestra satisfecho y agradecido con la intervención.

\section{Discusión y conclusiones.}

En base a los resultados obtenidos, tanto en las escalas psicométricas como en las informaciones cualitativas realizadas por el participante, la principal conclusión es que la intervención realizada ha resultado eficaz para lograr los objetivos de la demanda de consulta.

En cuanto a la hipótesis inicial, según la cual el eje central de la intervención era el circulo vicioso generado en torno a las palpitaciones, si bien a mitad del tratamiento podían considerarse resueltos la mayoría de síntomas de ansiedad, para que la intervención resultase de utilidad a largo plazo debían trabajarse también las creencias que estaban en la base de dicha ansiedad, e incrementar los recursos de afrontamiento del participante para prevenir recaídas, incrementar la autoconfianza y dar lugar al crecimiento personal del cliente.

Por ello, la intervención derivó de forma natural a trabajar otras fuentes de insatisfacción no necesariamente vinculadas al deporte o la vida laboral.

No obstante, el hecho de que hayan persistido determinadas preocupaciones sobre la salud y la forma física (hablamos de una persona cuya actividad laboral se dirige a la promoción de hábitos de vida saludable, incluyendo el deporte, la alimentación, etc.) puede tomarse como una señal de que no es necesario tanto el transformar las creencias del cliente, como adaptarlas de forma funcional a los cambios o evoluciones de su ciclo vital, pues no es el contenido de dichas creencias lo erróneo en este caso, sino su intensidad y las consecuencias de aplicarlas en exceso.

Ryff y Keyes (1995) definen el bienestar psicológico como "la condición mental y emocional que establece la actividad psíquica adecuada de un individuo con respecto a su modelo individual y a la forma de adaptarse a las necesidades intrínsecas y extrínsecas del contexto físico y social. Se vincula este bienestar con las creencias que dirigen las actividades de los seres humanos y el sentido otorgado a las vivencias pasadas. Asociado a esto, está el bienestar con tener vivencias tanto comprendidas emocionalmente como de manera cognitiva de modo beneficiosa, con la habilidad de evitar algunas estimadas como incorrectas y con la capacidad de acomodarse a diferentes situaciones con tolerancia" (p.189); Nardone y Salvini (2006), indican que se trata en último término de cambiar el sistema perceptivo reactivo del cliente y su construcción de la realidad. 
La intervención realizada es un ejemplo de cómo partiendo del modelo general de intervención de la terapia breve estratégica pueden insertarse otras técnicas que permitan que el cliente llegue a modificar su sistema perceptivo reactivo, para asumir una interpretación diferente de su realidad y romper con el círculo vicioso que está manteniendo y ampliando su problema. Por tanto, el caso presentado permite mostrar cómo la integración de técnicas de diferentes escuelas o áreas de intervención puede ser beneficiosa para garantizar la solución de dicho problema y ampliar sus beneficios aportando desarrollo y crecimiento personal al cliente, sin necesidad de que la intervención deba ser excesivamente dilatada en el tiempo o en el número de sesiones. No obstante, deben tomarse en cuenta las limitaciones que supone extrapolar la metodología empleada a otros casos, pues al tratarse de un diseño de caso único, la intervención debería ser adaptada a los objetivos y demandas de cada caso.

\section{Referencias}

Beyebach, M. y Herrero de Vega, M (2016). 200 tareas en terapia breve. Barcelona: Herder.

Buceta, J.M. (1998). Psicología del entrenamiento deportivo. Madrid: Dykinson.

Cano-Vindel, A. y Miguel-Tobal, J.J. (2012) Sociedad Española para el estudio de la ansiedad y el estrés. Recuperado de http:// www.ansiedadyestres.org/test_de_ansiedad

Clark, D. y Beck, A. (2012). Terapia cognitiva para los trastornos de ansiedad. Bilbao: Desclée de Brouwer.

Colegio Oficial de Psicólogos de España (1998). Perfiles profesionales del psicólogo. Madrid: COP-E.

Díaz, D., Rodríguez-Cavajal, R., Blanco, A., Moreno-Jiménez, B., Gallardo, I., Valle, C. y Van-Dierendonck, D. (2006). Adaptación española de las escalas de bienestar psicológico de Ryff. Psicothema, 18, (3), 572 - 577.

Locke, E. y Latham (1984). Establecimiento de Objetivos. En Weinberg y Gould. Fundamentos de Psicología del deporte y el Ejercicio físico. Barcelona: Ariel.

Mitchell, B. (2017). Manual MSD. Recuperado de http://www.msdmanuals.com

Nadal, M. Elosua, R. y Mont, L. (2016) La práctica deportiva de resistencia como factor de riesgo para la fibrilación auricular. Cuadernos de estimulación cardiaca,1, (1),15-18.

Nardone, G. (2003). Más allá del miedo. Barcelona: Paidós.

Nardone, G. (2004). No hay noche que no vea el día. El tratamiento de los ataques de pánico. Barcelona: Herder.

Nardone G. y Salvini, A. (2006). El diálogo estratégico.Barcelona: RBA.

Ryff, C. (1989). Happiness is Everything, or Is It? Exploration on the Meaning of Psychological Well-Being. Journal of Personality and Social Psychology. 57 (6),1069-1081.

Ryff, C., y Keyes, L. (1995). The structure of psychological well-being revisited. Journal of Personality and Social Psychology, 69 (4), 719-727

Watzlawick, P. y Nardone, G. (1993). El arte del cambio. Barcelona: Herder.

Watzlawick, P. y Nardone, G. (2014). Terapia Breve Estratégica. Barcelona:Paidós.

Weinberg, R. y Gould, D. (1995). Fundamentos de psicología del deporte y del ejercicio físico. Barcelona: Ariel. 